\title{
SPICE Mutator Model for Transforming Memristor into Meminductor
}

\author{
Hui Wang, ${ }^{1}$ Xin Wang, ${ }^{2}$ Chuandong Li, ${ }^{2}$ and Ling Chen ${ }^{2}$ \\ ${ }^{1}$ College of Mathematics Science, Chongqing Normal University, Chongqing 401331, China \\ ${ }^{2}$ College of Computer Science, Chongqing University, Chongqing 400044, China
}

Correspondence should be addressed to Chuandong Li; licd@cqu.edu.cn

Received 23 October 2012; Accepted 4 December 2012

Academic Editor: Xiaodi Li

Copyright (C) 2013 Hui Wang et al. This is an open access article distributed under the Creative Commons Attribution License, which permits unrestricted use, distribution, and reproduction in any medium, provided the original work is properly cited.

The memristor (resistor with memory), as the fourth fundamental circuit element, is a nonvolatile nanoelectronic device and holds great promise for VLSI applications. It was suggested that the meminductor (ML, inductor with memory) circuit can be built by memristor emulators. This paper further addresses the transformation mechanism in terms of constitutive relation from the memristor to the meminductor and then designs an MR-ML mutator to achieve MR-ML transformation. We also present the mutator's SPICE model and analyze the simulation results.

\section{Introduction}

The memristor (a contraction for memory resistor) was believed to be the fourth fundamental two-terminal passive element, besides the resistor, the capacitor, and the inductor. The element was firstly postulated by Chua [1] to characterize the relationship between the charge and the flux linkage for the sake of the completeness of the circuit theory. However, this pioneer work had not attracted the researchers' attention until 2008. Strukov et al., at Hewlett Packard, announced the first experimental design of memristor-two $\mathrm{TiO}_{2}$ layers sandwiched between two platinum electrodes [2]. This work has been causing a tremendously increased interest in the field of the theory and applications of the memristor. Progressive potential applications of memristor cover many important areas, such as digital memory logic, analog circuits, and neural synapse [1,3-7].

The memristor is a special case of a more general memristive system which was defined by Chua and Kang [8]. In 2009, the memcapacitatative and meminductive systems were also formulated in the spirit of memristive systems. As the special types of the memcapacitive and meminductive systems, memcapacitor and meminductor [9] also attract interest of the scientists. Combining with memristive systems, these elements will open up much new and unexpected functionality in the field of electronics [10].
Recently, several emulator models for meminductor and memcapacitor were built $[11,12]$. However, these emulator models are complicated for circuit implementation. To conquer this problem, we present a design method of mutator for MR-ML transformation, which makes the circuit realization and theoretical analysis much more convenient and effective. This method can apply easily to build the mutator for MR$\mathrm{MC}$ transformation. In the next section, we will analyze the constitutive relation of meminductor via CR transforming method. Then, the idea for transforming MR to $\mathrm{ML}$ is proposed in Section 3. Based on the SPICE model of the memristor, the mutator model for meminductor will be designed in Section 4, and then simulation and analysis results are demonstrated in Section 5. Finally, the conclusions are drawn in Section 6.

\section{Constitutive Relation of Meminductor}

As discussed in [13], a network element can be defined axiomatically by its constitutive relation, which does not depend on the element interaction with the surrounding networks. The constitutive relation of meminductor is

$$
f(\rho, q)=0,
$$




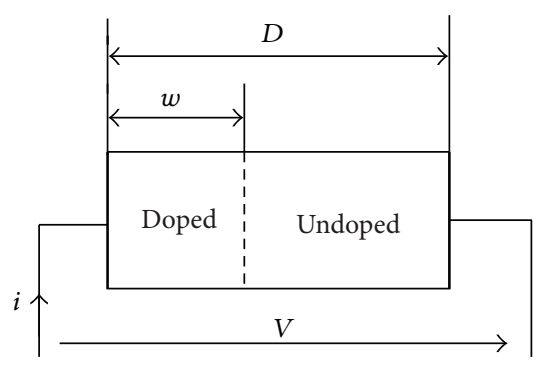

FIGURE 1: The variable resistor model.

where $\rho, q$ are defined by

$$
\rho(t)=\int_{-\infty}^{t} \phi(\sigma) d \sigma, \quad q(t)=\int_{-\infty}^{t} i(\sigma) d \sigma .
$$

Assuming that constitutive relation (1) can be depicted as a single-valued function $h$ of charge, namely,

$$
\rho(t)=h(q(t))
$$

where $h$ is a nonlinear function, then the current-controlled meminductor's constitutive relation can be rewritten as

$$
\phi(t)=L_{M}(q) i(t)
$$

where $L_{M}(q)=d h(q) / d q \mid Q$ is the small-signal meminductance defined at the operating $Q$.

\section{Transformation from Memristor to Meminductor}

In this paper, we use the memristor model proposed in [14] to demonstrate the theoretical possibility of transforming the memristor into the meminductor.

The memristor models presented in $[2,3,14]$ are shown in Figure 1. The total resistance of the memristor, $R_{\mathrm{MEM}}$, is a sum of the resistance of the doped and undoped regions, namely,

$$
R_{\mathrm{MEM}}(x)=R_{\mathrm{ON}} \frac{w(t)}{D}+R_{\mathrm{OFF}}\left(1-\frac{w(t)}{D}\right),
$$

where $w$ and $D$ are the width of the doped region and the total length of the $\mathrm{TiO}_{2}$ layer, respectively, and $R_{\mathrm{ON}}$ and $R_{\mathrm{OFF}}$ are the limit values of the memristor resistance for $w=D$ and $w=0$. is

The corresponding port equation $(\mathrm{PE})$ of the memristor

$$
v(t)=\left[R_{\mathrm{ON}} \frac{w(t)}{D}+R_{\mathrm{OFF}}\left(1-\frac{w(t)}{D}\right)\right] i(t),
$$

where $w(t)$ is the state variable about the length of the doped region in the thin film. $d w(t) / d t$ is proportional to the current passing through the memristor. The model presented in [12] used the window function which is the Joglekar function $\left(f(x)=1-(2 x-1)^{2 p}\right)$ to model nonlinear dopant drift. But in order to analyze conveniently, we define the window function as $f(x)=1$, while choosing the Joglekar function [14] with the parameter $p=1$ in model design.

The speed of the movement of the boundary between the doped and undoped regions depends on the resistance of the doped area, on the passing current, and on the other factors according to the states equation $[1,14]$,

$$
\frac{d w(t)}{d t}=u_{v} \frac{R_{\mathrm{ON}}}{D} i(t) .
$$

Therefore $w$ is a function of charge

$$
w(t)=u_{v} \frac{R_{\mathrm{ON}}}{D} q(t) .
$$

Based on (8) and $0 \leq w(t) \leq D$, the condition for the memristor regime is $0 \leq q(t) \leq D^{2} / u_{v} R_{\mathrm{ON}}$. Substituting (8) into (5), one can observe that the memristor is acting like a variable resistor with the resistivity $M(q)$ which is a function with respect to charge, namely,

$$
M(q)=R_{\mathrm{OFF}}-\left(R_{\mathrm{OFF}}-R_{\mathrm{ON}}\right) \frac{u_{v} R_{\mathrm{ON}}}{D^{2}} q(t) .
$$

From the definition of the memristor $d \phi=M(q) d q$, it follows that

$$
\phi(t)=R_{\mathrm{OFF}} q(t)-\left(R_{\mathrm{OFF}}-R_{\mathrm{ON}}\right) \frac{u_{v} R_{\mathrm{ON}}}{D^{2}} q^{2}(t) .
$$

As we know, the constitutive relation of the memristor has the same form with (10). Arguing similarly with [15, 16], we now transform the port equation into the constitutive relation. For this purpose, we denote

$$
r=\left(R_{\mathrm{OFF}}-R_{\mathrm{ON}}\right) \frac{u_{v} R_{\mathrm{ON}}}{D^{2}} .
$$

It follows from (2) that

$$
\rho=\int_{-\infty}^{t}\left[R_{\mathrm{OFF}} q(\sigma)-r q^{2}(\sigma)\right] d \sigma .
$$

Therefore,

$$
L_{M}(q)=\frac{d h(q)}{d q}=\frac{d\left(\int_{-\infty}^{t}\left[R_{\mathrm{OFF}} q(t)-r q^{2}(t)\right] d(t)\right)}{d q} .
$$

By the discussion above, one can see that the meminductance is a function of charge if $q \neq 0$, and otherwise $h(q)=0$.

Hence, we have obtained the constitutive relation of meminductor $\rho(q)(12)$ and the meminductance (13) $L_{M}(q)$ upon expanding the constitutive relation of the memristor.

\section{Mutator from MR into ML}

4.1. Characterization of the $M R-M L$ Mutator. The mutator is an active, two-port linear network for transforming one type of nonlinear element into another type. There are three types of mutators - an L-R mutator, a C-R mutator, and an L-C mutator-which have been realized [17]. In this subsection, 


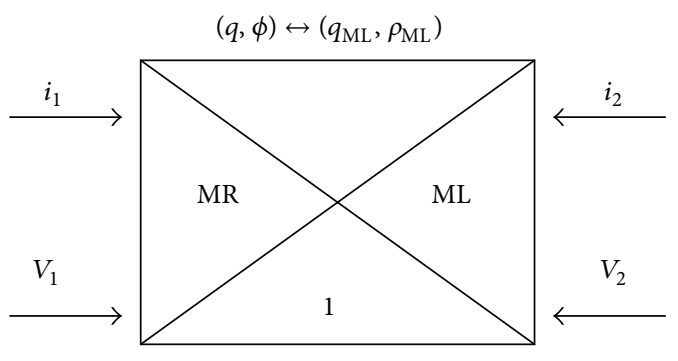

FIgURE 2: Characterization of MR-ML mutator.

we introduce an MR-ML mutator which is firstly proposed in $[15,18]$. It is characterized by the property that if a nonlinear memristor with a $v-i$ curve $\gamma$ is connected across port 2 of this element, the resulting one port (seen across port 1 ) becomes a nonlinear inductor in the sense that it can be characterized by a $\phi-i$ curve which is identical to the original $v$-i curve $\gamma$. The basic principle of a mutator is as shown in Figure 2.

Inspired by the idea presented in [15], we design a mutator for simulating meminductor through the memristors. The constitutive relations of MR and ML can be accomplished by the following linear transformation of the coordinates $\varphi=k_{x} \rho_{\mathrm{ML}}, q=k_{y} q_{\mathrm{ML}}$, where $k_{x}$ and $k_{y}$ are real constants. Their values depend on the way the concrete mutator is implemented. The time-domain differentiation of the linear transformation yields the following: $v_{2}=k_{x}(d / d t) v_{1}, i_{2}=$ $k_{y} i_{1}$. These equations are written in the operator form by means of the transmission matrix $T$ [15]. So we can have the following matrix as the transmission matrix:

$$
\left[\begin{array}{l}
v_{1} \\
i_{1}
\end{array}\right]=\left[\begin{array}{cc}
s k_{x} & 0 \\
0 & -k_{y}
\end{array}\right]\left[\begin{array}{l}
v_{2} \\
i_{2}
\end{array}\right] .
$$

4.2. Demonstration by Example. In this subsection, we apply a sinusoidal current source defined by

$$
\begin{gathered}
i(t)=A \sin (\omega t) \quad t \geq 0, \\
i(t)=0 \quad t<0
\end{gathered}
$$

across this memristor, where $A=1$ and $\omega=1$. One can calculate the corresponding charge (assuming the initial charge $q_{0}=q(0)=0$ )

$$
q(t)=\int_{0}^{t} A \sin (\omega \tau) d \tau=1-\cos t, \quad t \geq 0 .
$$

Substituting (16) into (10) yields the corresponding flux

$$
\phi(t)=R_{\mathrm{OFF}}(1-\cos t)-r(1-\cos t)^{2} .
$$

Substituting (17) into (12) then yields the corresponding TIF (integral of magnetic flux)

$$
\rho=\left(R_{\mathrm{OFF}}-\frac{3 r}{2}\right) t+\left(2 r-R_{\mathrm{OFF}}^{2}\right) \sin (\omega t)-r \sin (2 \omega t) .
$$

In a period $[-T / 2, T / 2]$, it follows from (16) that

$$
t=\arccos (1-q), \quad 0 \leq q \leq 2 .
$$

This together with (18) implies that

$$
\begin{aligned}
\rho(q)= & \left(R_{\mathrm{OFF}}-\frac{3 r}{2}\right) \arccos (1-q) \\
& +\left(2 r-R_{\mathrm{OFF}}^{2}\right) \sin \{\omega[\arccos (1-q)]\} \\
& -r \sin \{2 \omega[\arccos (1-q)]\} .
\end{aligned}
$$

Note that the constitutive relation holds the following condition which is one of the fingerprints of the meminductors: $\rho(q)=0$, for $q=0$.

For the existence of higher-order derivative of $\rho(q)$, one can rewrite (20) in the form of the Taylor series [11]:

$$
\rho=h(q)=\sum_{k=1}^{\infty} \gamma_{k} q^{k}
$$

And therefore the meminductance $L_{M}(q)$ is rewritten as

$$
\begin{aligned}
L_{M}(q)=\frac{1}{\sqrt{1-(1-q)^{2}}}\{ & R_{\mathrm{OFF}}-\frac{3 r}{2}+\left(2 r-R_{\mathrm{OFF}}^{2}\right) \\
& \times \omega \cos [\omega \arccos (1-q)] \\
& -2 \omega r \cos [\omega \arccos (1-q)]\}
\end{aligned}
$$

or, in form of the Taylor series,

$$
L_{M}(q)=\gamma_{1}+\sum_{k=2}^{\infty} \gamma_{k} q^{k}
$$

From (23) one observes that if $\gamma_{k}=0$, for $k>1$, the meminductance is independent of the circuit variables; namely, the meminductor behaves as a linear inductor. In other words, the memory effect is described by the remaining terms of the Taylor series just for $k>1$. From the constitutive relation $\rho(q)$ of meminductor, (15), and the meminductance $L_{M}(q)$, (13), one observes that the proposed results for meminductor by expanding the constitutive relation of the memristor are well consistent with those in [15].

4.3. Realization of MR-ML Mutator Model. In this subsection, we present a mutator to transform MR to ML inspired by the idea in $[15,18,19]$. The PSpice model of the mutator is based on the following steps.

(i) The terminal voltage of mutator is sensed and led to a cascade of two time-domain integrations in order to get the flux $(\phi)$ and the $\operatorname{TIF}(\rho)$.

(ii) Based on the knowledge of the charge and TIF, the meminductance is computed from (13).

(iii) Based on the knowledge of the $L_{M}(q)$ and $\phi$, the current $i$ of the meminductor mutator can be computed as follows:

$$
i(t)=\frac{1}{L_{M}(q)} \varphi(t)=\left(\gamma_{1}+\sum_{k=2}^{\infty} \gamma_{k} q^{k}\right)\left(\varphi(0)+\int_{0}^{t} v(\sigma) d \sigma\right) .
$$




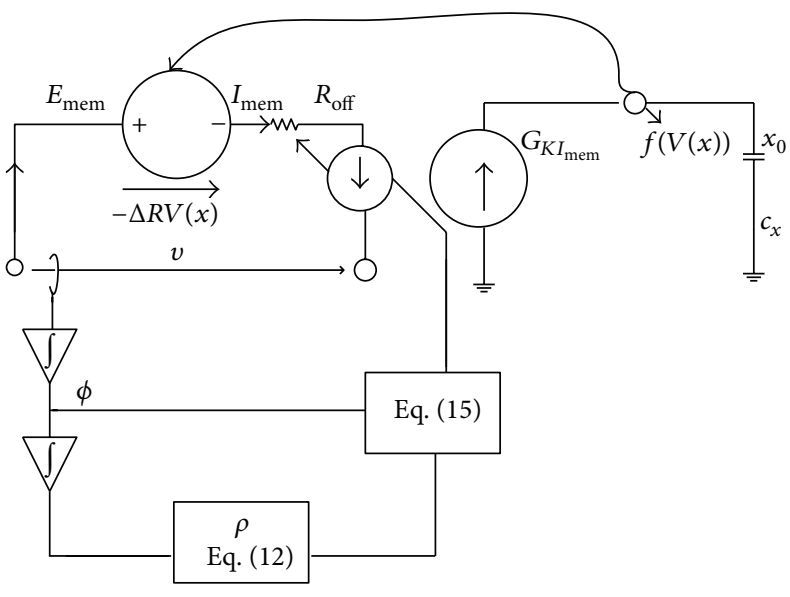

FIGURE 3: SPICE model of the mutator.

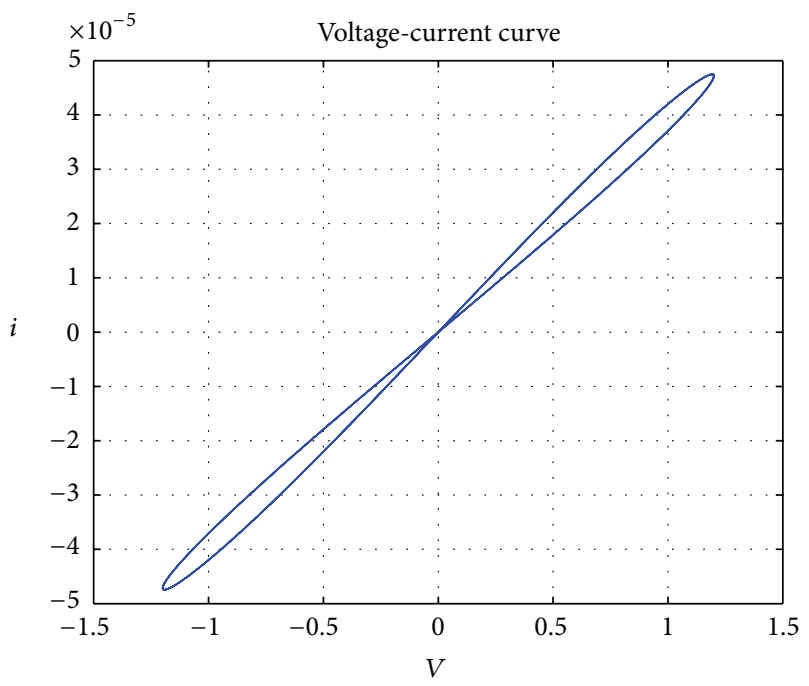

FIgURE 4: The $V$-I characteristics of memristor.

The PSpice model of the mutator proposed in this paper is shown in Figure 3.

\section{Simulation Results}

To verify the effectiveness of the proposed mutator, SPICE simulation is designed and simulation results with series waveforms demonstrate its performance. The run time is set as $2 \mathrm{~s}$. We connect the sinusoidal current source as the excitation of the circuit. The voltage and the current flow through the memristor as the excitation of e port 1.

Consider the memristor model shown in Figure 1 with the parameters $R_{\mathrm{ON}}=100, R_{\mathrm{OFF}}=16 \mathrm{~K}, R_{\text {init }}=11 \mathrm{k}$, $D \approx 10 \mathrm{~nm}$, and $u_{v} \approx 10^{-14} \mathrm{~m}^{2} \mathrm{~s}^{-1} \mathrm{~V}^{-1}$. As used in [12], the window function is chosen as a smoother Joglekar function with the parameter $p=1$. We use the sinusoidal voltage source, with the parameter of the peak amplitude $V_{a}=1.4 \mathrm{~V}$,

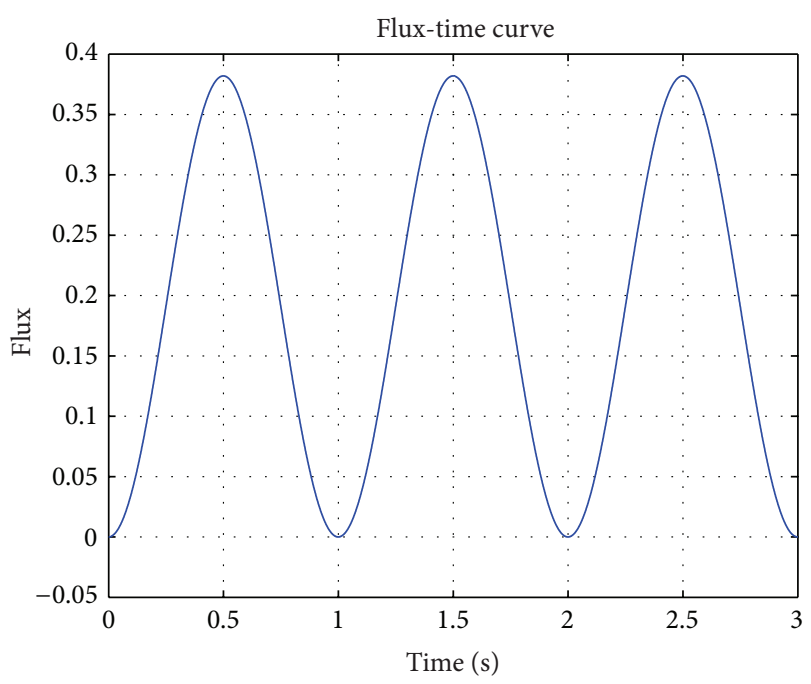

(a)

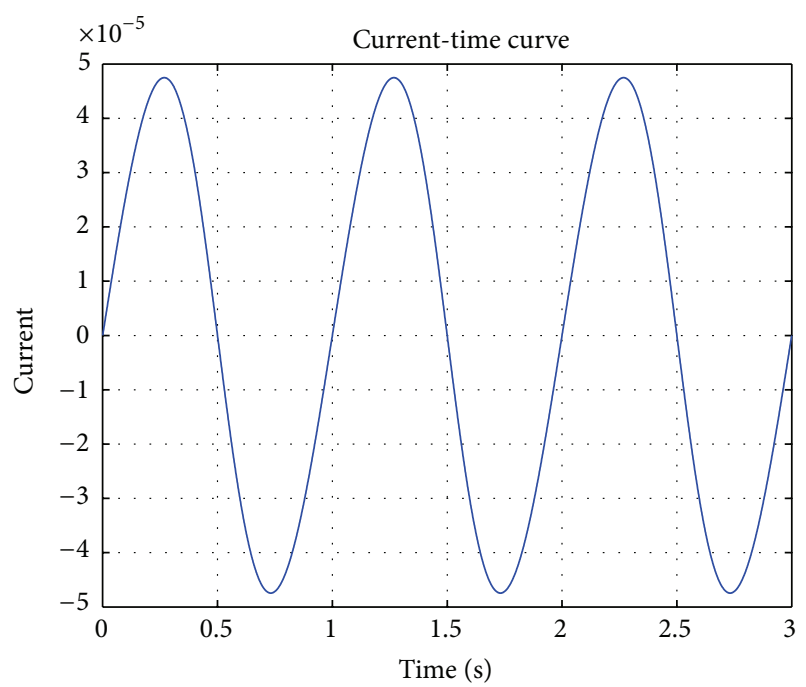

(b)

Figure 5: (a) Flux of meminductor; (b) current of meminductor.

and the frequency freq $=0.325 \mathrm{HZ}$. The $V-I$ characteristic of memristor is presented in Figure 4.

The $\phi(t)$ curve and the $i(t)$ curve of port 1, which are used to characterize the property of the meminductor, are shown in Figures 5(a) and 5(b). Figure 5(a) represents the flux of the meminductor, and Figure 5(b) represents the current, respectively. Figure 6 shows the meminductance $L_{\mathrm{ML}}\left(L_{\mathrm{ML}}=\right.$ $\phi(t) / i(t))$ curve.

\section{Conclusions}

We have demonstrated that the current-controlled meminductor can be transformed into memristor through the constitutive relation expanding. The mutator presented here provides a true MR-ML transformation. Using the procedure mentioned above, the memristor-to-memcapacitor mutator can also be easily designed. Together with the recently 


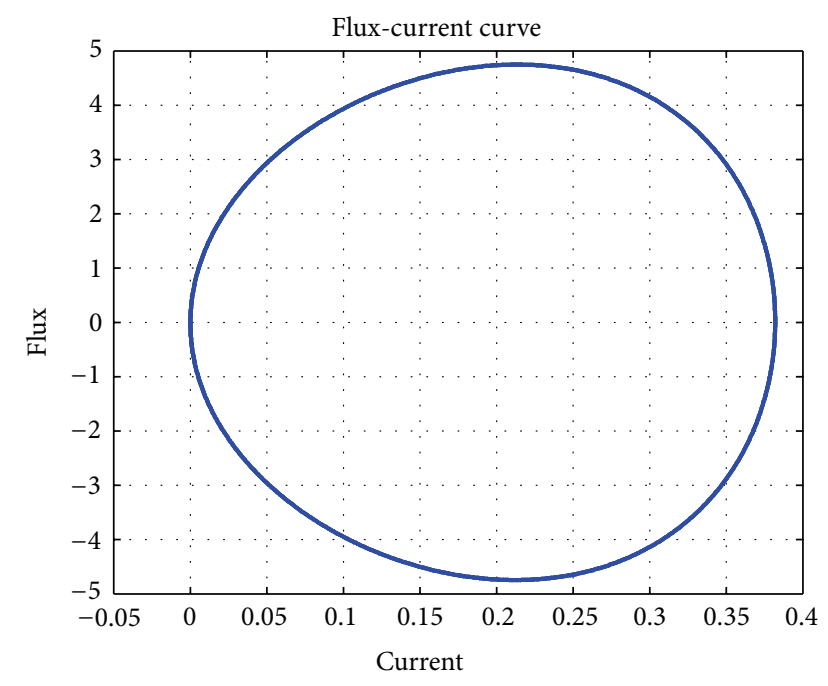

Figure 6: The meminductance curve for sinusoidal source.

reported mutator, these circuits serve as useful tools for analyzing these new memory elements.

\section{Acknowledgments}

This work was supported in part by the National Natural Science Foundation of China under Grant 60974020, 60972155, the Natural Science Foundation of Chongqing under Grant CSTC2009BB2305, the Fundamental Research Funds for the Central Universities (Grant no. XDJK2010C023), and the National Science Foundation for Post-doctoral Scientists of China (Grant no. CPSF20100470116).

\section{References}

[1] L. O. Chua, "Memristor-the missing circuit element," IEEE Transaction on Circuit and System, vol. 18, no. 5, pp. 507-519, 1971.

[2] D. B. Strukov, G. S. Snider, D. R. Stewart, and R. S. Williams, "The missing memristor found," Nature, vol. 453, pp. 80-83, 2008.

[3] R. S. Williams, "How we found the missing circuit element," IEEE Spectrum, pp. 1-11, 2008.

[4] G. S. Rose, "Overview: memristive devices, circuits and systems," in Proceedings of the IEEE International Symposium on Circuits and Systems (ISCAS '10), pp. 1955-1958, Paris, France, June 2010.

[5] X. F. Hu, S. K. Duan, L. D. Wang, and X. F. Liao, "Memristive crossbar array with applications in image processing," Science China, vol. 41, no. 4, pp. 500-512, 2011.

[6] Q. Xia, W. Robinett, M. W. Cumbie et al., "Memristor-CMOS hybrid integrated circuits for reconfigurable logic," Nano Letters, vol. 9, no. 10, pp. 3640-3645, 2009.

[7] S. H. Jo, T. Chang, I. Ebong, B. B. Bhadviya, P. Mazumder, and W. Lu, "Nanoscale memristor device as synapse in neuromorphic systems," Nano Letters, vol. 10, no. 4, pp. 1297-1301, 2010.

[8] L. O. Chua and S. M. Kang, "Memristive devices and systems," Proceedings of the IEEE, vol. 64, no. 2, pp. 209-223, 1976.
[9] M. D. Ventra, Y. V. Pershin, and L. O. Chua, "Circuit elements with memory: memristors, memcapacitors, and meminductors," Proceedings of the IEEE, vol. 97, no. 10, pp. 1717-1724, 2009.

[10] M. D. Ventra, Y. V. Pershin, and L. O. Chua, "Putting memory into circuit elements: memristors, memcapacitors, and meminductors," Proceedings of the IEEE, vol. 97, no. 8, pp. 1371-1372, 2009.

[11] D. Biolek, V. Biolková, and Z. Kolka, "SPICE modeling of meminductor based on its constitutive relation," in Proceedings of the 10th WSEAS International Conference on Instrumentation, Measurement, Circuits and Systems (IMCAS '11), pp. 76-79, March 2011.

[12] D. Biolek, Z. Biolek, and V. Biolkova, "SPICE modeling of memcapacitor," Electronics Letters, vol. 46, no. 7, pp. 520-522, 2010.

[13] L. O. Chua, "Nonlinear circuit foundations for nanodevicespart I: the four-element torus," Proceedings of the IEEE, vol. 91, no. 11, pp. 1830-1859, 2003.

[14] Z. Biolek, D. Biolek, and V. Biolková, "SPICE model of memristor with nonlinear dopant drift," Radioengineering, vol. 18, no. 2, pp. 210-214, 2009.

[15] D. Biolek, V. Biolková, and Z. Kolka, "Mutators simulating memcapacitors and meminductors," in Proceedings of the Asia Pacific Conference on Circuit and System (APCCAS '10), pp. 800803, Kuala Lumpur, Malaysia, December 2010.

[16] M. Mahvash and A. C. Parker, "A memristor SPICE model for designing memristor circuits," in Proceedings of the 53rd IEEE International Midwest Symposium on Circuits and Systems (MWSCAS '10), pp. 989-992, Seattle, Wash, USA, August 2010.

[17] L. O. Chua, "Synthesis of new nonlinear network elements," Proceedings of the IEEE, vol. 56, no. 8, pp. 1325-1340, 1968.

[18] Y. V. Pershin and M. D. Ventra, "Memristive circuits simulate memcapacatiors and meminductors," Electronics Letters, vol. 46, no. 7, pp. 517-518, 2010.

[19] D. Biolek and V. Biolkova, "Mutator for transforming memristor into memcapacitor," Electronics Letters, vol. 46, no. 21, pp. 14281429, 2010. 


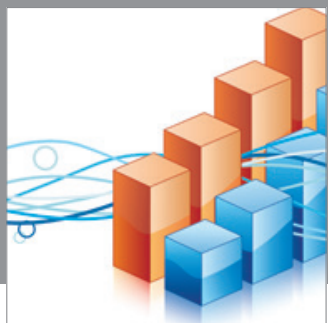

Advances in

Operations Research

mansans

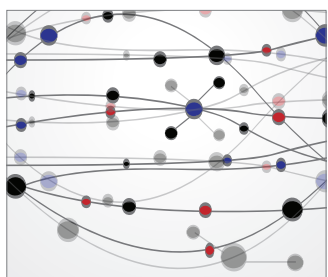

The Scientific World Journal
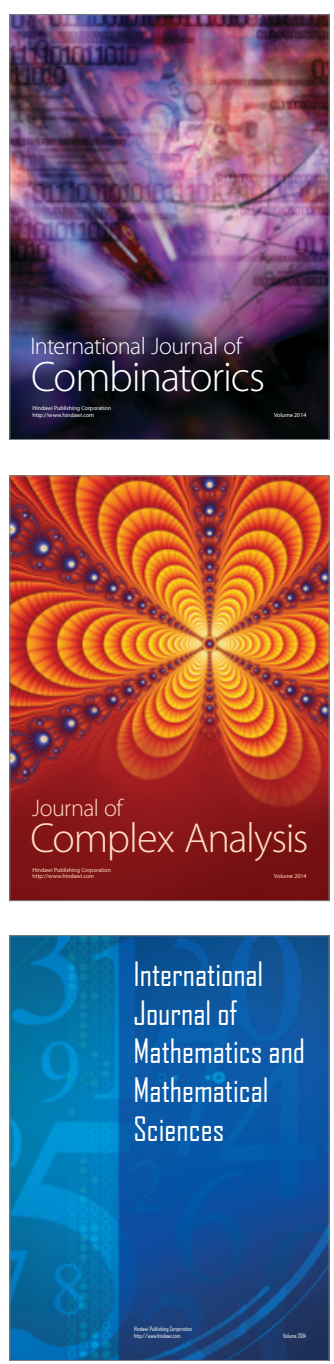
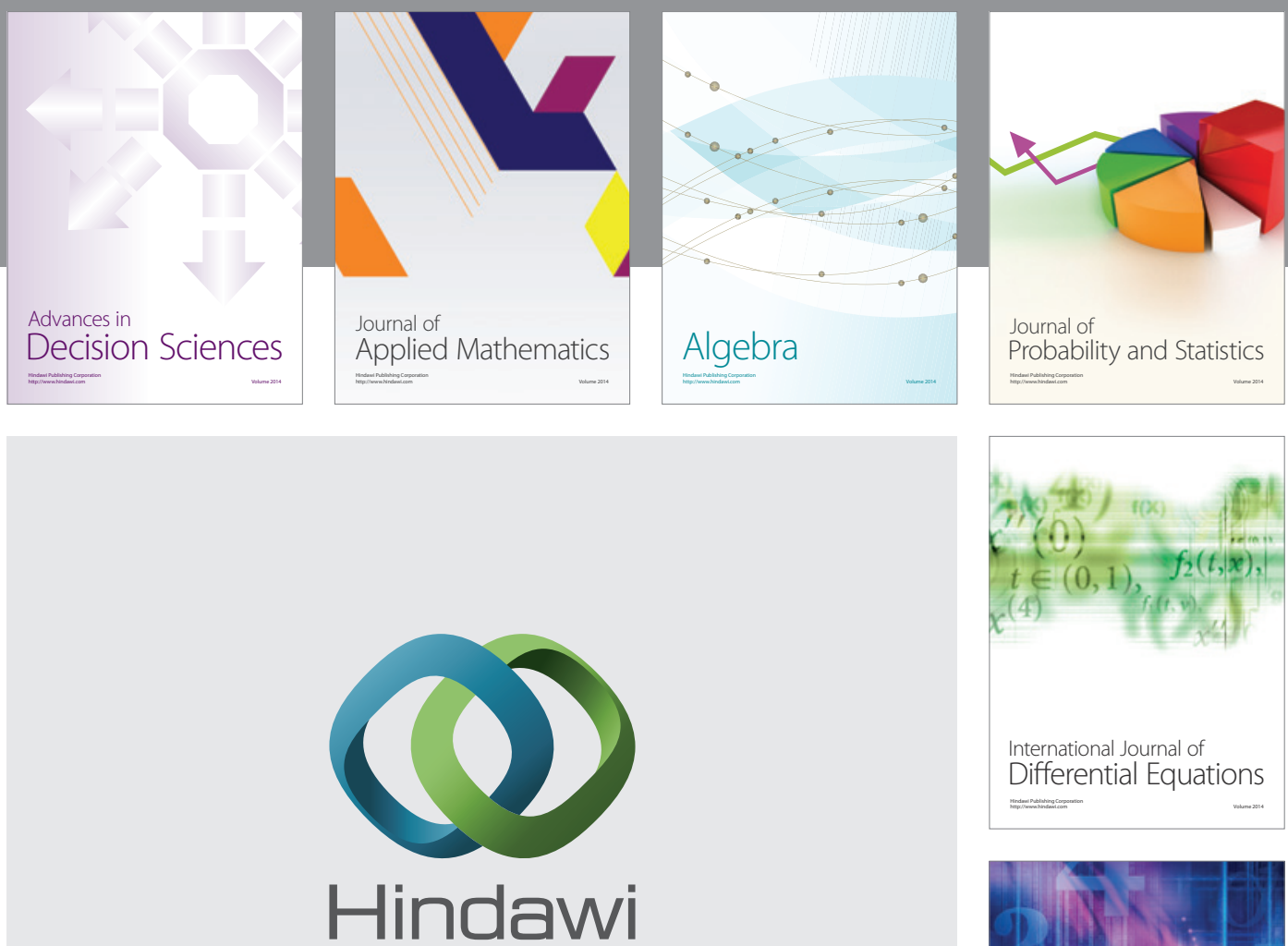

Submit your manuscripts at http://www.hindawi.com
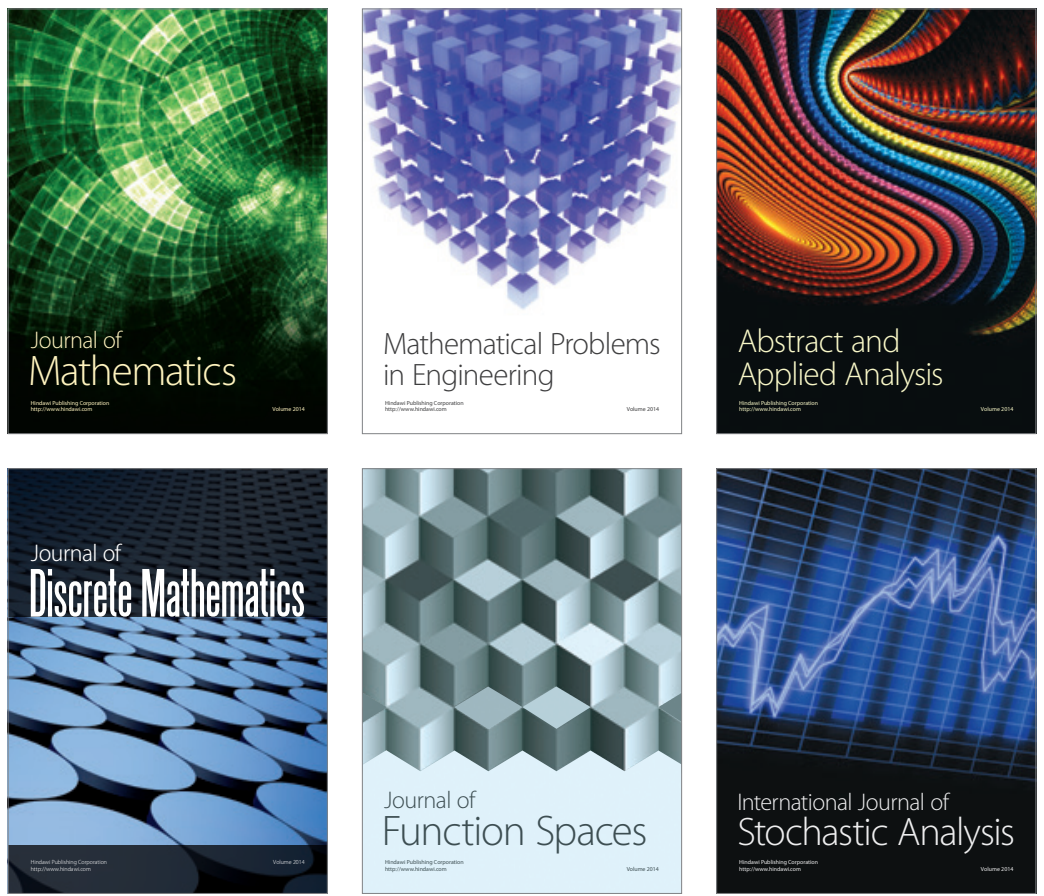

Journal of

Function Spaces

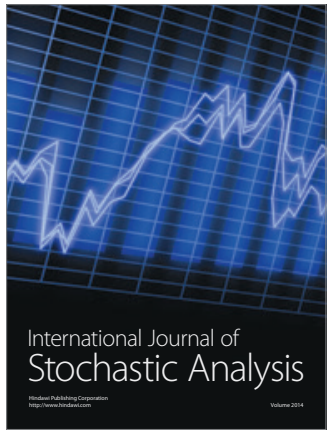

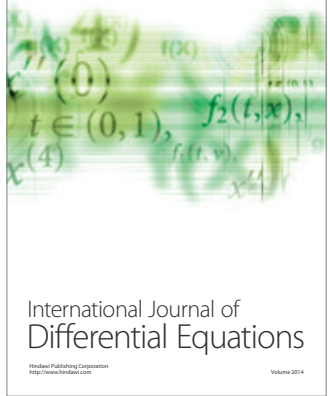
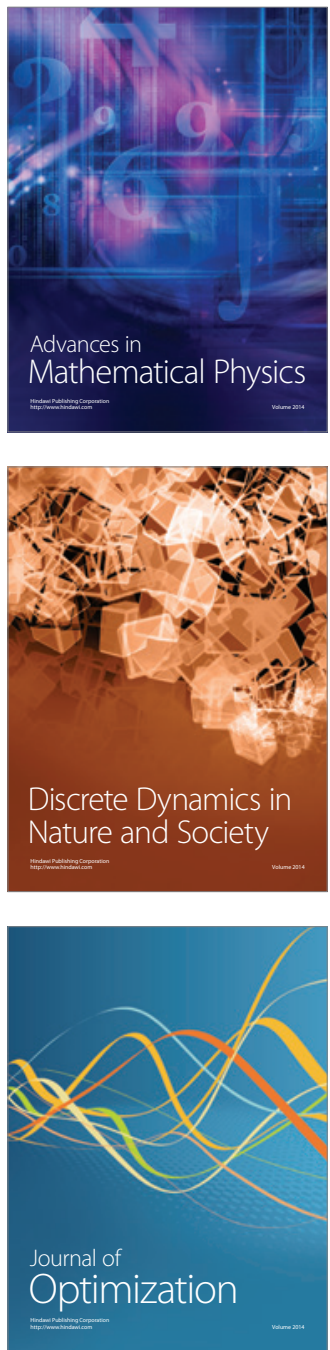